\title{
Nuclear parton distribution functions from neutrino deep inelastic scattering
}

\author{
I. Schienbein, ${ }^{1,2, *}$ J. Y. Yu, ${ }^{1, \dagger}$ C. Keppel, ${ }^{3,4, \$}$ J. G. Morfín, ${ }^{5, \S}$ F. Olness, ${ }^{1,7, \|}$ and J. F. Owens ${ }^{1,6, \pi}$ \\ ${ }^{1}$ Southern Methodist University, Dallas, Texas 75206, USA \\ ${ }^{2}$ Laboratoire de Physique Subatomique et de Cosmologie, Université Joseph Fourier Grenoble 1, CNRS/IN2P3, \\ Institut National Polytechnique de Grenoble, 53 Avenue des Martyrs, 38026 Grenoble, France \\ ${ }^{3}$ Thomas Jefferson National Accelerator Facility, Newport News, Virginia 23602, USA \\ ${ }^{4}$ Hampton University, Hampton, Virginia, 23668, USA \\ ${ }^{5}$ Fermilab, Batavia, Illinois 60510, USA \\ ${ }^{6}$ Florida State University, Tallahassee, Florida 32306-4350, USA \\ ${ }^{7}$ Theoretical Physics Division, Physics Department, CERN, CH 1211 Geneva 23, Switzerland
}

(Received 12 November 2007; published 14 March 2008)

\begin{abstract}
We study nuclear effects in charged current deep inelastic neutrino-iron scattering in the framework of a $\chi^{2}$ analysis of parton distribution functions (PDFs). We extract a set of iron PDFs and show that under reasonable assumptions it is possible to constrain the valence, light sea, and strange quark distributions. Our iron PDFs are used to compute $x_{B j}$-dependent and $Q^{2}$-dependent nuclear correction factors for iron structure functions which are required in global analyses of free nucleon PDFs. We compare our results with nuclear correction factors from neutrino-nucleus scattering models and correction factors for $\ell^{ \pm}$-iron scattering. We find that, except for very high $x_{B j}$, our correction factors differ in both shape and magnitude from the correction factors of the models and charged-lepton scattering.
\end{abstract}

DOI: 10.1103/PhysRevD.77.054013

PACS numbers: $12.38 .-\mathrm{t}, 13.15 .+\mathrm{g}, 13.60 .-\mathrm{r}, 24.85 .+\mathrm{p}$

\section{INTRODUCTION}

The high statistics measurements of neutrino deeply inelastic scattering (DIS) on heavy nuclear targets has generated significant interest in the literature since these measurements provide valuable information for global fits of parton distribution functions (PDFs) [1]. The use of nuclear targets is unavoidable due to the weak nature of the neutrino interactions, and this complicates the extraction of free nucleon PDFs because model-dependent corrections must be applied to the data.

Additionally, these same data are also useful for extracting the nuclear parton distribution functions (NPDFs); for such an analysis, no nuclear correction factors are required. Because of the limited statistics available for individual nuclear targets with a given atomic number $A$ the standard approach is to model the $A$-dependence of the fit parameters, and then combine the data sets for many different target materials in the global analysis [2-7]. However, the high statistics $\mathrm{NuTeV}$ neutrino-iron cross section data ( $>2000$ points) offer the possibility to investigate the viability of a dedicated determination of iron PDFs [8].

With this motivation, we will perform a fit to the $\mathrm{NuTeV}$ neutrino-iron data and extract the corresponding iron

\footnotetext{
*schien@1psc.in2p3.fr

†yu@physics.smu.edu

*keppel@jlab.org

\$morfin@fnal.gov

" olness@smu.edu

Towens@hep.fsu.edu
}

PDFs. Since we are studying iron alone and will not (at present) combine the data with measurements on different target materials, we need not make any assumptions about the nuclear corrections; this sidesteps a number of difficulties [9-11].

While this approach has the advantage that we do not need to model the $A$-dependence, it has the drawback that the data from just one experiment will not be sufficient to constrain all the parton distributions. Therefore, other assumptions must enter the analysis. The theoretical framework will roughly follow the CTEQ6 analysis of free proton PDFs [12]; this will be discussed in Sec. II.

In Sec. III we present the results of our analysis, and compare with nuclear PDFs from the literature. In Sec. IV we extract the nuclear correction factors from our iron PDFs and compare with a SLAC/NMC parametrization taken from the $\ell^{ \pm}$-Fe DIS process [13] and also with the parametrization by Kulagin and Petti [14,15]. Finally, we summarize our results and conclusions in Sec. V.

\section{THEORETICAL FRAMEWORK}

\section{A. Basic formalism}

For our PDF analysis, we will use the general features of the QCD-improved parton model and the $\chi^{2}$ analyses as outlined in Ref. [12]. Here, we will focus on the issues specific to our study of NuTeV neutrino-iron data in terms of nuclear parton distribution functions. We adopt the framework of the recent CTEQ6 analysis of proton PDFs where the input distributions at the scale $Q_{0}=1.3 \mathrm{GeV}$ 
are parametrized as [12]

$$
x f_{i}\left(x, Q_{0}\right)= \begin{cases}A_{0} x^{A_{1}}(1-x)^{A_{2}} e^{A_{3} x}\left(1+e^{A_{4}} x\right)^{A_{5}} & : i=u_{v}, d_{v}, g, \bar{u}+\bar{d}, s, \bar{s}, \\ A_{0} x^{A_{1}}(1-x)^{A_{2}}+\left(1+A_{3} x\right)(1-x)^{A_{4}} & : i=\bar{d} / \bar{u},\end{cases}
$$

where $u_{v}$ and $d_{v}$ are the up- and down-quark valence distributions, $\bar{u}, \bar{d}, s, \bar{s}$ are the up, down, strange, and anti-strange-sea distributions, and $g$ is the gluon. Furthermore, the $f_{i}=f_{i}^{p / A}$ denote parton distributions of bound protons in the nucleus $A$, and the variable $0 \leq x \leq$ $A$ is defined as $x:=A x_{A}$ where $x_{A}=Q^{2} / 2 p_{A} \cdot q$ is the usual Bjorken variable formed out of the four-momenta of the nucleus and the exchanged boson. Equation (1) is designed for $0 \leq x \leq 1$ and we here neglect ${ }^{1}$ the distributions at $x>1$. Note that the condition $f_{i}(x>1, Q)=0$ is preserved by the DGLAP evolution and has the effect that the evolution equations and sum rules for the $f_{i}^{p / A}$ are the same as in the free-proton case. ${ }^{2}$

The PDFs for a nucleus $(A, Z)$ are constructed as

$$
f_{i}^{A}(x, Q)=\frac{Z}{A} f_{i}^{p / A}(x, Q)+\frac{(A-Z)}{A} f_{i}^{n / A}(x, Q),
$$

where we relate the distributions inside a bound neutron, $f_{i}^{n / A}(x, Q)$, to the ones in a proton by assuming isospin symmetry. Similarly, the nuclear structure functions are given by

$$
F_{i}^{A}(x, Q)=\frac{Z}{A} F_{i}^{p / A}(x, Q)+\frac{(A-Z)}{A} F_{i}^{n / A}(x, Q)
$$

such that they can be computed in next-to-leading order (NLO) as convolutions of the nuclear PDFs with the conventional Wilson coefficients, i.e., generically

$$
F_{i}^{A}(x, Q)=\sum_{k} C_{i k} \otimes f_{k}^{A} .
$$

In order to take into account heavy quark mass effects we calculate the relevant structure functions in the ACOT scheme $[17,18]$ in NLO QCD [19]. Finally, the differential cross section for charged current (anti)neutrino-nucleus scattering is given in terms of three structure functions:

$$
\begin{aligned}
\frac{d^{2} \sigma^{(-)} A}{d x d y}= & \frac{G^{2} M E}{\pi}\left[\left(1-y-\frac{M x y}{2 E}\right) F_{2}^{\stackrel{(-)}{\nu} A}+\frac{y^{2}}{2} 2 x F_{1}^{(-)} A\right. \\
& \left. \pm y\left(1-\frac{y}{2}\right) x F_{3}^{(-)} A\right]
\end{aligned}
$$

\footnotetext{
${ }^{1}$ While the nuclear PDFs can be finite for $x>1$, the magnitude of the PDFs in this region is negligible for the purposes of the present study (cf., Refs. [2-6]).

${ }^{2}$ While the quark number and momentum sum rules for the nuclear case are satisfied as in the proton, there is no requirement that the momentum fractions carried by the PDF flavors be the same. A recent analysis at low $Q^{2}$ found the Cornwall-Norton moments to be the same in iron as in deuterium formed from a free proton and a free neutron to within $3 \%$ [16].
}

where the "+" ("-") sign refers to neutrino (antineutrino) scattering and where $G$ is the Fermi constant, $M$ the nucleon mass, and $E$ the energy of the incoming lepton (in the laboratory frame).

\section{B. Constraints on PDFs}

We briefly discuss which combinations of PDFs can be constrained by the neutrino-iron data. For simplicity, we restrict ourselves to leading order, neglect heavy quark mass effects (as well as the associated production thresholds), and assume a diagonal Cabibbo-KobayashiMaskawa (CKM) matrix. ${ }^{3}$ The neutrino-iron structure functions are given by (suppressing the dependence on $x$ and $Q^{2}$ ):

$$
\begin{gathered}
F_{1}^{\nu A}=d^{A}+s^{A}+\bar{u}^{A}+\bar{c}^{A}+\ldots, \\
F_{2}^{\nu A}=2 x F_{1}^{\nu A}, \\
F_{3}^{\nu A}=2\left[d^{A}+s^{A}-\bar{u}^{A}-\bar{c}^{A}+\ldots\right] .
\end{gathered}
$$

The structure functions for antineutrino scattering are obtained by exchanging the quark and antiquark PDFs in the corresponding neutrino structure functions:

$$
\begin{aligned}
& F_{1,2}^{\bar{\nu} A}=+F_{1,2}^{\nu A}[q \leftrightarrow \bar{q}], \\
& F_{3}^{\bar{\nu} A}=-F_{3}^{\nu A}[q \leftrightarrow \bar{q}] .
\end{aligned}
$$

Explicitly this gives

$$
\begin{gathered}
F_{1}^{\bar{\nu} A}=u^{A}+c^{A}+\bar{d}^{A}+\bar{s}^{A}+\ldots, \\
F_{2}^{\bar{\nu} A}=2 x F_{1}^{\bar{\nu} A}, \\
F_{3}^{\bar{\nu} A}=2\left[u^{A}+c^{A}-\bar{d}^{A}-\bar{s}^{A}+\ldots\right] .
\end{gathered}
$$

It is instructive to compare this with the parton model expressions for the structure function $F_{2}$ in $l^{ \pm} A$ scattering, where $l^{ \pm}$denotes a charged lepton:

$$
\begin{aligned}
\frac{1}{x} F_{2}^{l A}= & \frac{4}{9}\left(u^{A}+\bar{u}^{A}\right)+\frac{1}{9}\left(d^{A}+\bar{d}^{A}\right)+\frac{1}{9}\left(s^{A}+\bar{s}^{A}\right) \\
& +\frac{4}{9}\left(c^{A}+\bar{c}^{A}\right)+\ldots
\end{aligned}
$$

Using the Callan-Gross relations in Eqs. (7) and (12), and neglecting the proton mass, the differential cross section Eq. (5) can be simplified in the form

\footnotetext{
${ }^{3}$ All these effects are properly included in our calculations.
} 


$$
d \sigma \propto\left(1-y+y^{2} / 2\right) F_{2}^{(-)} A+y\left(1-\frac{y}{2}\right) x F_{3}^{(\stackrel{(-)}{\nu A}}
$$

with the limiting cases:

$$
d \sigma \rightarrow \begin{cases}\frac{1}{2} F_{2}^{\nu A} \pm \frac{(-)}{2} x F_{3}^{(-)} A & (\text { for } y \rightarrow 1) \\ F_{2}^{(-)} A & (\text { for } y \rightarrow 0)\end{cases}
$$

The latter form of $d \sigma$ shows that the (anti)neutrino cross section data naturally encodes information on the four structure function combinations $F_{2}^{(-)} A+x F_{3}^{\nu} A$ and $F_{2}^{(-)} A$ in separate regions of the phase space.

If we assume ${ }^{4} s^{A}=\bar{s}^{A}$ and $c^{A}=\bar{c}^{A}$, the structure functions $F_{2}^{\stackrel{(-)}{\nu}}$ constrain the valence distributions $d_{v}^{A}=d^{A}-$ $\bar{d}^{A}, u_{v}^{A}=u^{A}-\bar{u}^{A}$ and the flavor-symmetric sea $\Sigma^{A}:=$ $\bar{u}^{A}+\bar{d}^{A}+\bar{s}^{A}+\bar{c}^{A}+\ldots$ via the relations:

$$
\begin{gathered}
\frac{1}{x} F_{2}^{\nu A}=2\left[d_{v}^{A}+\Sigma^{A}\right], \\
\frac{1}{x} F_{2}^{\bar{\nu} A}=2\left[u_{v}^{A}+\Sigma^{A}\right] .
\end{gathered}
$$

Furthermore, we have

$$
\begin{aligned}
& \frac{1}{x} F_{2}^{\nu A}+F_{3}^{\nu A}=4\left(d^{A}+s^{A}\right), \\
& \frac{1}{x} F_{2}^{\bar{\nu} A}-F_{3}^{\bar{\nu} A}=4\left(\bar{d}^{A}+\bar{s}^{A}\right) .
\end{aligned}
$$

Since we constrain the strange distribution utilizing the dimuon data, ${ }^{5}$ the latter two structure functions are useful to separately extract the $d^{A}$ and $\bar{d}^{A}$ distributions.

For an isoscalar nucleus we encounter further simplifications. In this case, $u^{A}=d^{A}$ and $\bar{u}^{A}=\bar{d}^{A}=: \bar{q}^{A}$ which implies $u_{v}^{A}=d_{v}^{A}=: v^{A}$. Hence, the independent quark distributions are $\left\{v^{A}, \bar{q}^{A}, s^{A}=\bar{s}^{A}, c^{A}=\bar{c}^{A}, \ldots\right\}$. It is instructive to introduce the parameter $\Delta:=1 / 2-Z / A$ which describes the degree of nonisoscalarity. This allows us to write the PDFs in a way which makes deviations from isoscalarity manifest:

$$
\begin{aligned}
& u_{v}^{A}=v^{A}-\Delta\left[u_{v}^{p / A}-d_{v}^{p / A}\right], \\
& d_{v}^{A}=v^{A}+\Delta\left[u_{v}^{p / A}-d_{v}^{p / A}\right],
\end{aligned}
$$

\footnotetext{
${ }^{4}$ Note that these equations are known not to be exact as the DGLAP evolution equations at next-to-next-to-leading order (NNLO) generate an asymmetry even if one starts with $s=\bar{s}$ or $c=\bar{c}$ at some scale $Q^{2}$ [20]. However, these effects are tiny and far beyond the accuracy of our study.

${ }^{5}$ See Refs. [21-26] for details.
}

$$
\bar{u}^{A}=\bar{q}^{A}-\Delta\left[\bar{u}^{p / A}-\bar{d}^{p / A}\right]
$$

$$
\bar{d}^{A}=\bar{q}^{A}+\Delta\left[\bar{u}^{p / A}-\bar{d}^{p / A}\right]
$$

in terms of an averaged nuclear valence distribution $v^{A}=$ $\left(u_{v}^{p / A}+d_{v}^{p / A}\right) / 2$ and an averaged nuclear sea distribution $\bar{q}^{A}=\left(\bar{u}^{p / A}+\bar{d}^{p / A}\right) / 2$. Recall, $f_{i}^{p / A}$ represents the distribution for a bound proton in the nucleus $A$; hence, the nuclear effects are encoded in these terms. Notice that nonisoscalar targets $(\Delta \neq 0)$ therefore provide information on the difference between the valence distributions $\left(u_{v}^{p / A}-d_{v}^{p / A}\right)$ and the light quark sea distribution $\left(\bar{u}^{p / A}-\right.$ $\left.\bar{d}^{p / A}\right)$ in the nucleon. Unfortunately, the data are often corrected for nonisoscalar effects and this information is lost.

\section{Methodology}

The basic formalism described in the previous sections is implemented in a global PDF fitting package, but with the difference that no nuclear corrections are applied to the analyzed data; hence, the resulting PDFs are for a bound proton in an iron nucleus. The parametrization of Eq. (1) provides enough flexibility to describe current data sets entering a global analysis of free nucleon PDFs; given that the nuclear modifications of the $x$-shape appearing in this analysis are modest, this parametrization will also accommodate the iron PDFs.

Because the neutrino data alone do not have the power to constrain all of the PDF components, we will need to impose some minimal set of external constraints. For example, our results are rather insensitive to the details of the gluon distribution with respect to both the overall $\chi^{2}$ and also the effect on the quark distributions. The nuclear gluon distribution is very weakly constrained by present data, and a gluon PDF with small nuclear modifications has been found in the NLO analysis of Ref. [7]. We have therefore fixed the gluon input parameters to their free nucleon values. For the same reasons the gluon is not sensitive to this analysis, fixing the gluon will have minimal effect on our results. Furthermore, we have set the $\bar{d} / \bar{u}$ ratio to the free nucleon result assuming that the nuclear modifications to the down and up sea are similar such that they cancel in the ratio. This assumption is supported by Fig. 6 in Ref. [7].

Because we have limited the data set to a single heavy target (iron), the $\chi^{2}$ surface has some parameter directions which are relatively flat. To fully characterize the parameter space, we perform many "sample fits" starting from different initial conditions, and iterate these fits including/ excluding additional parameters. The result is a set of bands for fits of comparable quality $\left(\Delta \chi^{2} \sim 50\right.$ for 2691 data points) which provide an approximate measure of the constraining power of the data. 
TABLE I. Fits to NuTeV cross section and dimuon data.

\begin{tabular}{lcccccc}
\hline \hline Scheme & Cuts & Data & \# points & $\chi^{2}$ & $\chi^{2} / \mathrm{pts}$ & Name \\
\hline ACOT & $Q>1.3 \mathrm{GeV}$ & $\nu+\bar{\nu}$ & 2691 & 3678 & 1.37 & $\mathrm{~A}$ \\
& no $W_{\text {cut }}$ & $\nu$ & 1459 & 2139 & 1.47 & $\mathrm{~A} \nu$ \\
& & $\bar{\nu}$ & 1232 & 1430 & 1.16 & $\mathrm{~A} \bar{\nu}$ \\
ACOT & $Q>2 \mathrm{GeV}$ & $\nu+\bar{\nu}$ & 2310 & 3111 & 1.35 & $\mathrm{~A} 2$ \\
& $W>3.5 \mathrm{GeV}$ & $\nu$ & 1258 & 1783 & 1.42 & $\mathrm{~A} 2 \nu$ \\
& & $\bar{\nu}$ & 1052 & 1199 & 1.14 & $\mathrm{~A} 2 \bar{\nu}$ \\
$\overline{\mathrm{MS}}$ & $Q>1.3 \mathrm{GeV}$ & $\nu+\bar{\nu}$ & 2691 & 3732 & 1.39 & $\mathrm{M}$ \\
& no $W_{\text {cut }}$ & $\nu$ & 1459 & 2205 & 1.51 & $\mathrm{M} \nu$ \\
& & $\bar{\nu}$ & 1232 & 1419 & 1.15 & $\mathrm{M} \bar{\nu}$ \\
$\overline{\mathrm{MS}}$ & $Q>2 \mathrm{GeV}$ & $\nu+\bar{\nu}$ & 2310 & 3080 & 1.33 & $\mathrm{M} 2$ \\
& $W>3.5 \mathrm{GeV}$ & $\nu$ & 1258 & 1817 & 1.44 & $\mathrm{M} 2 \nu$ \\
& & $\bar{\nu}$ & 1052 & 1201 & 1.14 & $\mathrm{M} 2 \bar{\nu}$ \\
\hline \hline
\end{tabular}

\section{ANALYSIS OF IRON DATA}

\section{A. Iron data sets}

We determine iron PDFs using the recent NuTeV differential neutrino (1371/1170 data points) and antineutrino (1146/966 data points) DIS cross section data [8] where the quoted numbers of data points refer to the two different combinations of kinematic cuts introduced below. In addition, we include NuTeV/CCFR dimuon data (174 points) [21] which are sensitive to the strange quark content of the nucleon.

There are other measurements of neutrino-iron DIS available in the literature from the CCFR [27-30], CDHS [31], and CDHSW [32] collaborations; see, e.g., Ref. [33] for a review. There is also a wealth of charged lepton-iron DIS data including SLAC [34] and EMC $[35,36]{ }^{6}$ For the present study we limit our analysis to the NuTeV experiment alone; we will compare and contrast different experiments in a later study.

\section{B. Fit results}

The results of our fits to the $\mathrm{NuTeV}$ iron cross section and dimuon data are summarized in Table I. The cross section data have been corrected for QED radiation effects, and the nonisoscalarity of the iron target [37]; correspondingly, we have used $A=56, Z=28$ in Eqs. (2) and (3). ${ }^{7}$ Note, for an iron target the isoscalar correction factors are small and do not exceed the few-percent level. We have performed fits to the combined data as well as to the neutrino and antineutrino data sets separately. Furthermore, two different cuts in the kinematic plane have been examined: (a) $Q>1.3 \mathrm{GeV}$, no cut on the

\footnotetext{
${ }^{6} \mathrm{Cf}$. the Durham HEP databases for a complete listing: http:// www-spires.dur.ac.uk/hepdata/.

${ }^{7}$ We have checked that omitting the isoscalar correction factors and using $A=56, Z=26$ gives almost identical results.
}

hadronic invariant mass $W$ and (b) $Q>2 \mathrm{GeV}$ and $W>$ $3.5 \mathrm{GeV}$, cf., Table I. The NLO QCD calculation was performed in both the $\overline{\mathrm{MS}}$ and ACOT schemes. The ACOT scheme calculation takes into account the heavy quark mass effects, whereas the $\overline{\mathrm{MS}}$ scheme assumes massless partons. The dominant target mass effects have been incorporated $[38,39]{ }^{8}$

As noted above, we have found bands for each class of fits from which we have chosen central representatives. The $\chi^{2}$ values have been determined taking into account the full correlations of the data employing the effective $\chi^{2}$ function given in Eq. (B.5) of Ref. [12]. The numbers for the $\chi^{2} /$ pts are roughly on the order of 1.4 for both the ACOT and the $\overline{\mathrm{MS}}$ schemes. ${ }^{9}$ Furthermore, the fits to the antineutrino data have considerably better $\chi^{2}$ values; however, we will see below that this is at least partly due to the larger uncertainties of these data.

\section{PDF reference sets}

For the purposes of this study, we use two different reference sets of free-proton PDFs which we denote "Base-1" and "Base-2."

Since we focus on iron PDFs and the associated nuclear corrections, we need a base set of PDFs which are essentially free of any nuclear effects; this is the purpose of the Base-1 reference set [10]. Therefore, to extract the Base-1 PDFs we omit the CCFR and NuTeV data from our fit so that our base PDFs do not contain any large residual nuclear corrections. ${ }^{10}$ The absence of such nuclear effects will be important in Sec. IV when we extract the nuclear corrections factors.

The Base-2 PDFs are essentially the CTEQ6.1M PDFs with a modified strange PDF introduced to accommodate the NuTeV dimuon data. ${ }^{11}$ In the manner of the CTEQ6.1M PDFs, the Base-2 fit does not apply any deuteron corrections to the data; this is in contrast to the Base1 PDFs. Also, the Base-2 fit does include the CCFR data

\footnotetext{
${ }^{8}$ Target mass effects (TMC) are expected to be relevant at large Bjorken- $x$ or small momentum transfers $Q^{2}$ [39]. For issues of higher orders and higher twist, cf. Refs. [40-43].

${ }^{9}$ Fits to this same data neglecting the correlations between the errors and using the conventional $\chi^{2}$ function (cf. Eq. (B.1) in [12]), have smaller $\chi^{2} / \mathrm{pts} \simeq 1$. While the uncorrelated errors are larger, the extracted parameters are similar.

${ }^{10}$ We do retain the deuteron data as this has only a small correction over the central $x$-range, (cf. Sec. IVA) $[9,10]$. The deuteron correction has been applied in the Base-1 fit. Also, for the Drell-Yan $\mathrm{Cu}$ data (E605), the expected nuclear corrections in this kinematic range are small (a few percent) compared to the overall normalization uncertainty $(15 \%)$ and systematic error $(10 \%)$.

${ }^{11}$ These PDFs have been determined from a fit to the same data set as in the CTEQ6 analysis with the addition of the NuTeV dimuon data. The changes to the strange sea induce only minor changes to the other fit parameters; this has a minimal effect on the particular observables $\left(d \sigma, F_{2}\right)$ we examine in the present study.
} 


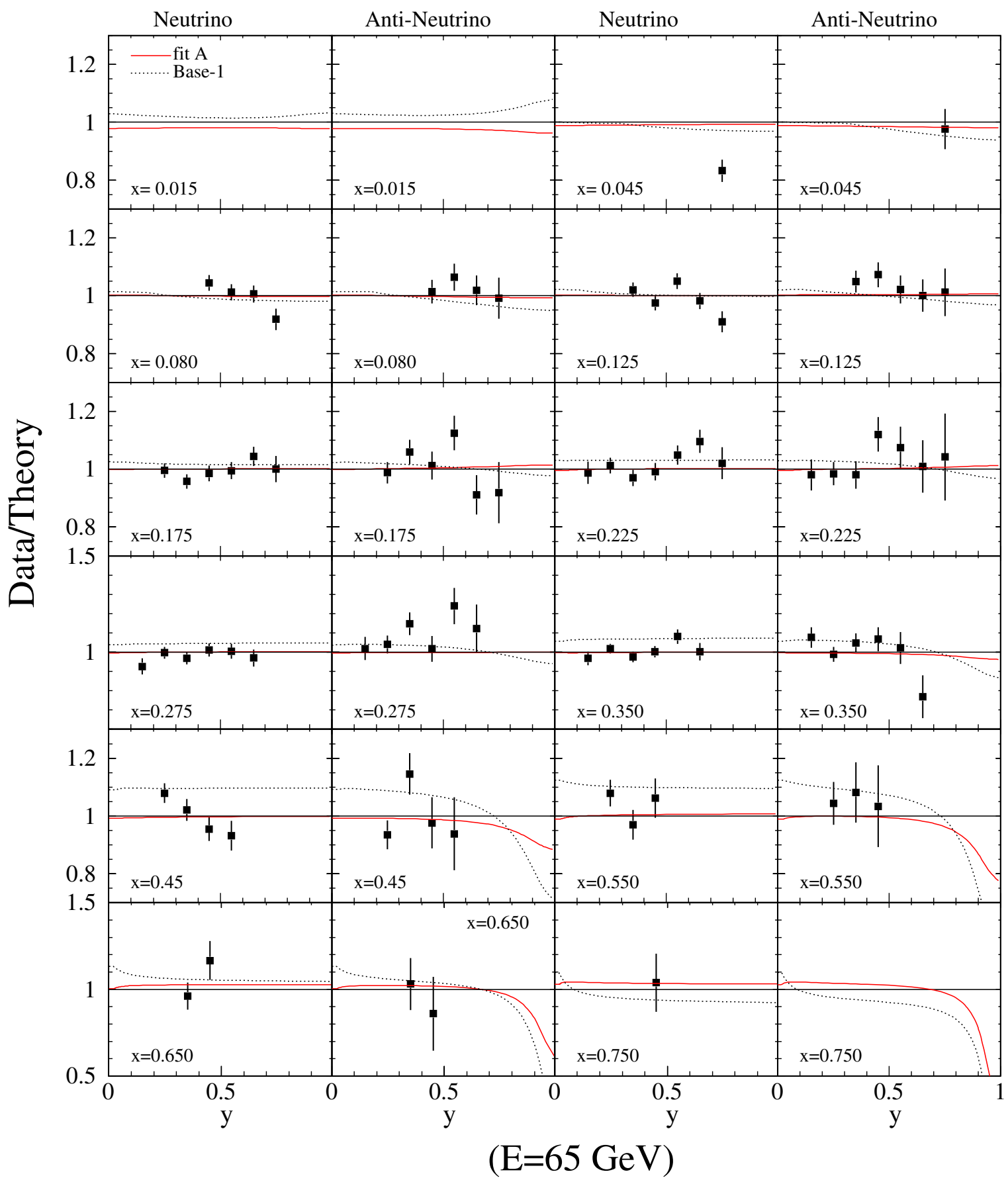

FIG. 1 (color online). Representative comparison of fit A2 to the NuTeV neutrino and antineutrino cross section data. Shown are the data points for various $x$-bins versus the inelasticity $y$ for an energy of $E=65 \mathrm{GeV}$ in a data-over-theory representation. For comparison, we also show results for the Base-1 PDFs (dotted lines) and the A fit (solid lines); the fit A2 imposes more stringent cuts on $Q>2 \mathrm{GeV}$ and $W>3.5 \mathrm{GeV}$.

that has been corrected to a free nucleon using chargedlepton correction factors; the Fermilab CCFR experiment is the predecessor of $\mathrm{NuTeV}$ with comparable statistics as those from $\mathrm{NuTeV}$ [30]. The CCFR results in the large- $x$ region $(x>0.4)$ are consistently lower than those from $\mathrm{NuTeV}$, and various sources contributing to the difference have been identified $[8,44]$. One-third of the discrepancy has been attributed to a miscalibration of the magnetic field map of the muon spectrometer, i.e., to the muon energy scale in the CCFR analysis. About another third comes from model differences (cross section model, muon, and hadron energy smearing models). A comparison of $\mathrm{NuTeV}$ and CCFR data can be found in Ref. [8].

By comparing the free-proton PDF Base-1 and Base-2 sets with the iron PDF sets of Table I, we can gauge the size 


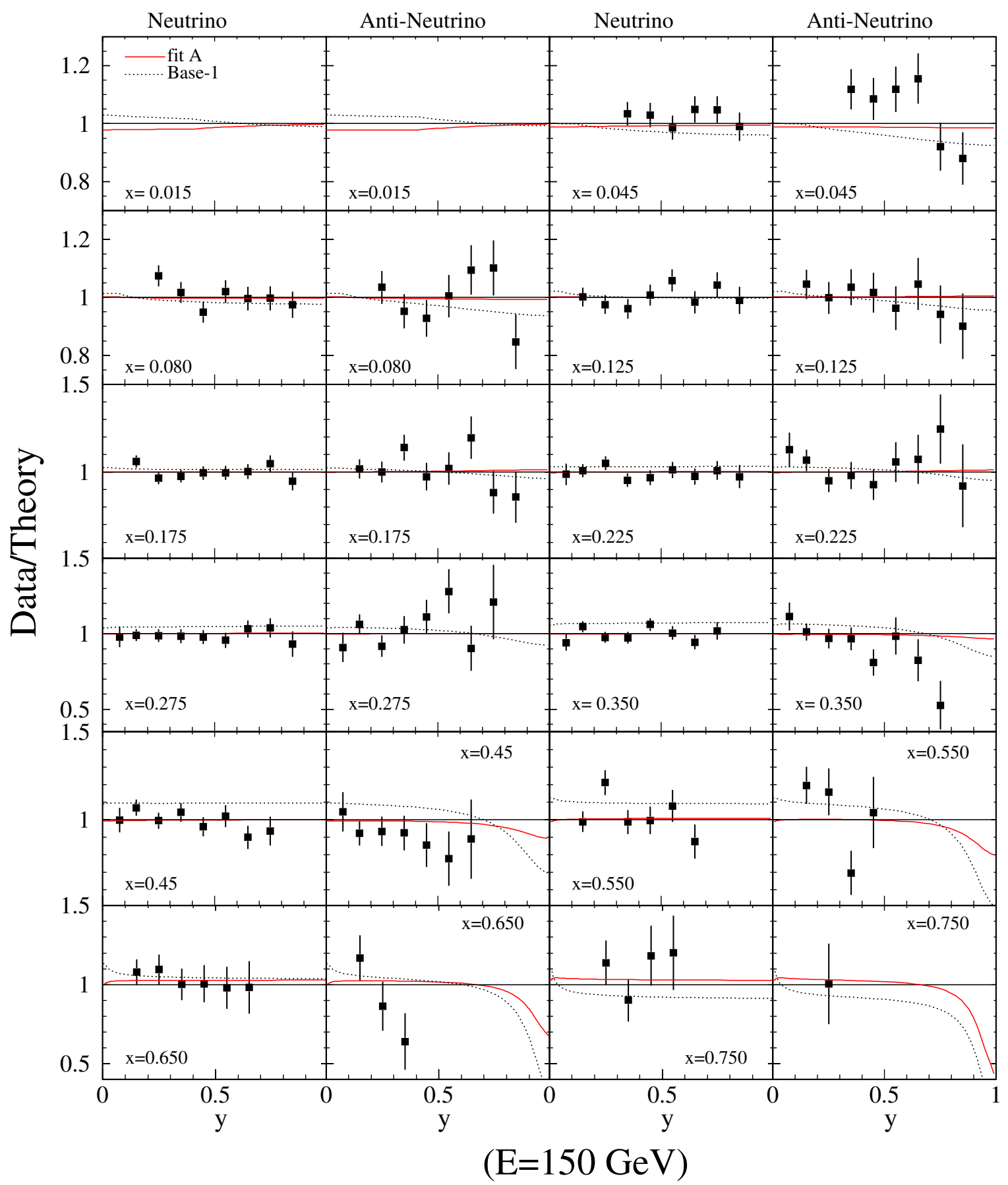

FIG. 2 (color online). The same as in Fig. 1 for a neutrino energy of $E=150 \mathrm{GeV}$.

of the nuclear effects. Furthermore, differences between observables using the Base-1, respectively, the Base-2 reference sets will indicate the uncertainty due to the choice of the free-proton PDF. ${ }^{12}$

\footnotetext{
${ }^{12}$ All results have been computed with both Base- 1 and Base-2 PDFs. Since the Base-2 PDFs use CCFR and NuTeV data, the resulting PDFs will depend on the nuclear corrections which we are trying to determine. Therefore, we will predominantly display the Base-1 PDFs for comparison in the following sections.
}

\section{Comparison of the fits with data}

The quality of our fits of Table I can also be observed directly in Figs. 1-3 where we compare the theoretical cross section $(1 / E) d^{2} \sigma / d x d y$ with a selection of the data. To be specific, we show all the data taken with beam energies $E=65,150$, and $245 \mathrm{GeV}$ which pass our kinematic cuts. The measurements are organized in bins of $x$ as a function of the inelasticity $y$ and cover the $x$-range $0.015 \leq x \leq 0.750$. The momentum transfers can be com- 


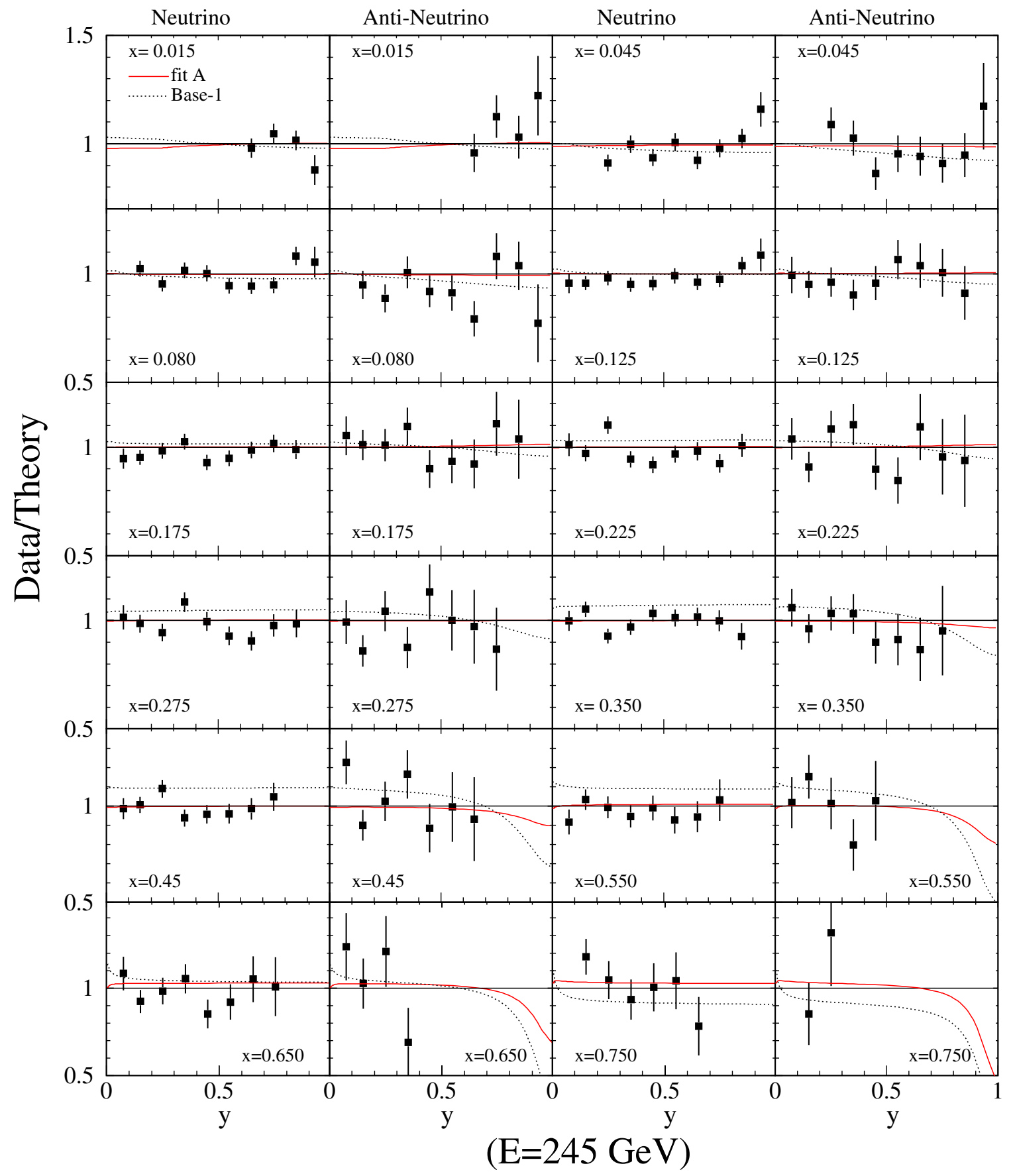

FIG. 3 (color online). The same as in Fig. 1 for a neutrino energy of $E=245 \mathrm{GeV}$.

puted using the relation $Q^{2}=2 M E x y$. We normalize these plots using the "A2" fit which implements the kinematic cuts $Q>2 \mathrm{GeV}$ and $W>3.5 \mathrm{GeV}$ (cf. Table I). We note that these are the cuts employed in the CTEQ6 analysis in order to reduce the sensitivity to target mass and higher twist effects. ${ }^{13}$

\footnotetext{
${ }^{13}$ Conversely, global analyses of nuclear PDFs tend to use looser kinematic cuts due to the lack of small- $x$ data and the interest in the very large- $x$ region.
}

The fit provides a good description of the data which are distributed around unity for most of the bins. For reference, the results of fit "A" (solid line) and Base-1 PDFs (dotted line) are shown as well. For fit A2, the effect of the $Q>$ $2 \mathrm{GeV}$ cut is to remove data at low $y$ in the small- $x$ region, and the $W>3.5 \mathrm{GeV}$ cut excludes low- $y$ data at large $x$. The effects of these cuts on the fit are visible by comparing the difference of the solid line (A) from unity (A2). For $x \gtrsim 0.045$, we observe minimal differences between the A and A2 fits, and conclude the effect of the kinematic cuts 
$(Q>2 \mathrm{GeV}$ and $W>3.5 \mathrm{GeV})$ are nominal in this region. In the lowest $x$ bin $(x \sim 0.015)$, much of the data is eliminated by the $Q>2 \mathrm{GeV}$ cut such that fit A2 is only constrained by a few data points at large $y$ for the higher neutrino energies, cf. Fig. 3. Since both, fit A and fit A2, have large uncertainties in this $x$-region the comparison of individual representatives is less significant - in particular at medium and low $y$ where no data points lie. In conclusion, we discern no relevant differences between the two classes of fits over the entire kinematic plane and will therefore mainly focus on fit $\mathrm{A} 2$ in the following sections.

\section{Comparison of the fits with reference PDFs}

The dotted curve in Figs. 1-3 shows the cross sections obtained with Base-1 free-proton PDFs, inserted into Eq. (2) to obtain "free iron" PDFs, divided by the cross sections computed with fit A2 PDFs. The Base-2 PDFs (not shown) yield similar results as we demonstrate in Sec. IV C. We expect the base PDFs will provide a poorer description of the data since the nuclear modifications are not taken into account; the deviations of these curves from unity indicate the size of the nuclear effects.

We observe that the Base- 1 results at small- $x(x \sim$ $[0.045-0.08])$ are generally below unity (the A2 fit) in the $y$ region of the data points implying an enhancement due to nuclear effects. As discussed above, the results in the lowest $x$ bin $(x=0.015)$ are less clear as the uncertainties are larger since the kinematic cuts remove much of the data. Nevertheless, we do not see a clear signal of shadowing in this region (cf., Fig. 3 at large $y$ ).

For intermediate $x \sim[0.125-0.175]$ the Base-1 (dotted line) results are very similar to fit A2. For larger $x \sim$ [0.225-0.65] we observe a suppression of the nuclear cross sections qualitatively similar to what is known from charged-lepton DIS. Finally, in the region $x \gtrsim 0.75$ the nuclear cross section is again enhanced - an effect usually attributed to the Fermi motion of the nucleons in the nucleus.

In conclusion, we observe the following pattern for the nuclear cross section compared to the free nucleon cross section: (i) enhancement for $x \geqslant 0.75$, (ii) suppression for $x \sim[0.225-0.65], \quad$ (iii) equality for $x \sim 0.125$, and (iv) slight enhancement for $x \sim[0.045-0.08]$. This is to be contrasted with the expectation from charged-lepton DIS with the well-known pattern: (i) enhancement for $x \gtrsim$ 0.75 (Fermi motion), (ii) suppression for $x \sim[0.3-0.8]$ (EMC effect), (iii) enhancement for $x \sim[0.06-0.3]$ (antishadowing), and (iv) suppression for $x \leqq 0.06$ (shadowing). Thus, for $x \geqslant 0.3$ our results are generally as expected. However, we find that the usual behavior at medium and small $x$ is modified. We will examine this further in the following sections.

\section{Iron PDFs}

Having established the quality of our fits, we now examine the nuclear (iron) parton distributions $f_{i}^{A}\left(x, Q^{2}\right)$ accord- ing to Eq. (2). Figure 4 shows the PDFs from fit A2 at our input scale $Q_{0}=m_{c}=1.3 \mathrm{GeV}$ versus $x$. For an almost isoscalar nucleus like iron the $u$ and $d$ distributions are very similar, see Eqs. (21)-(24). Therefore, we only show the
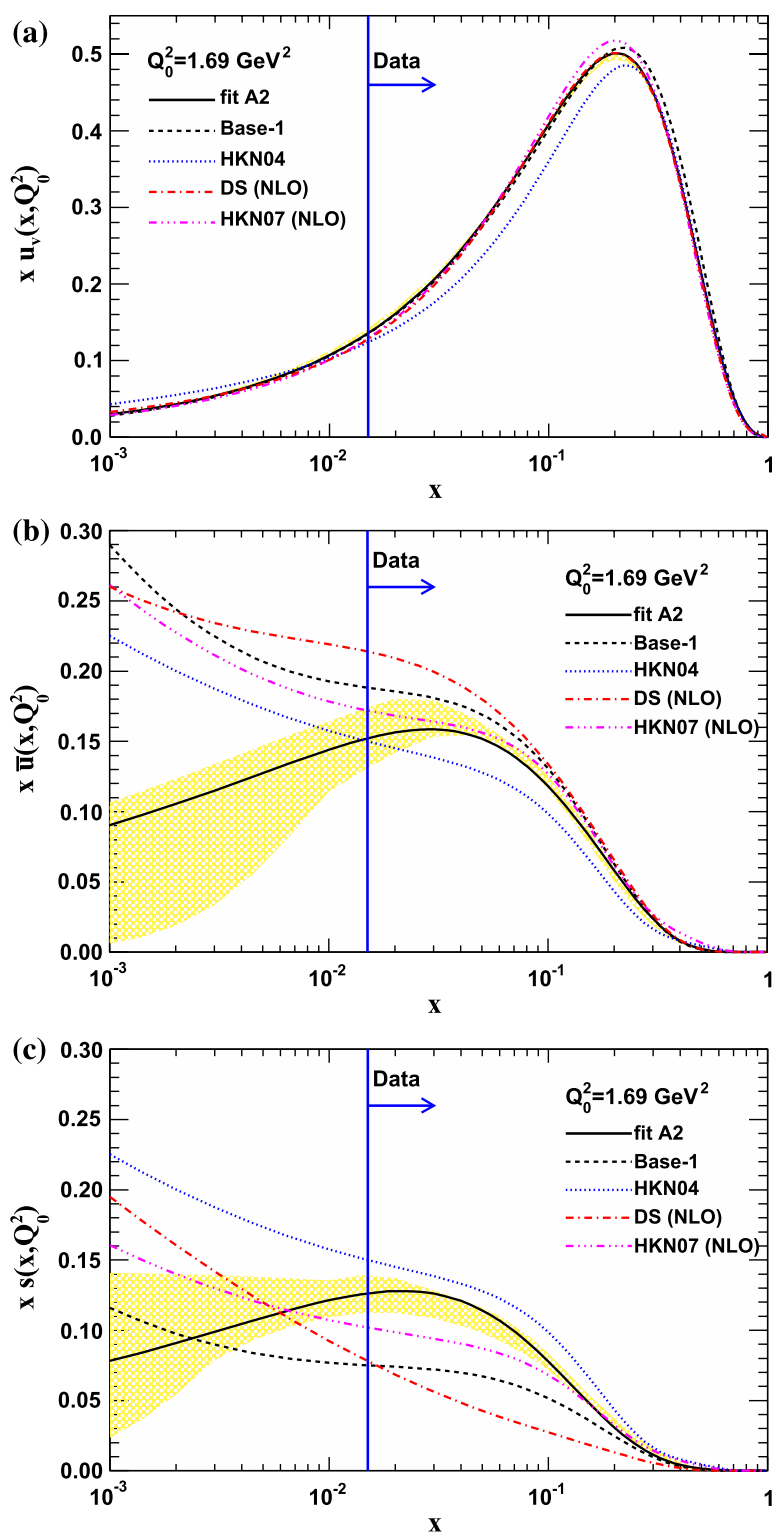

FIG. 4 (color online). Parton distributions for iron at our input scale $Q^{2}=1.69 \mathrm{GeV}^{2}$. Shown are the bands (in yellow) from fit A2 for the up-quark valence distribution (upper figure), the upquark sea (middle figure), and the strange quark sea (lower figure). The central PDF from fit A2 is shown by the solid line. The dashed lines depict parton distributions constructed according to Eq. (2) with $A=56$ and $Z=26$ using the Base- 1 free-proton PDFs. The dotted lines are the leading order HKN04 nuclear parton distributions [3], the dotted-dashed lines are the next-to-leading order HKN07 nuclear parton distributions [4], and the dot-dashed lines are the next-to-leading order distributions (DS) from Ref. [7]. The vertical line marks the lower limit of the data in the $x$ variable. 
$u_{v}$ and $\bar{u}$ partons, together with the strange sea. ${ }^{14}$ As explained above, the gluon distribution is very similar to the familiar CTEQ6M gluon at the input scale such that we do not show it here. In order to indicate the constraining power of the NuTeV data, the band of reasonable fits is depicted. The fits in this band were obtained (as outlined above) by varying the initial conditions and the number of free parameters to fully explore the solution space. All the fits shown in the band have $\chi^{2} / \mathrm{DOF}$ within 0.02 , which roughly corresponds to a range of $\Delta \chi^{2} \sim 50$ for the 2691 data points.

As can be seen in Fig. 4, the $u_{v}$ distribution [Fig. 4(a)] has a very narrow band across the entire $x$-range. The upand strange-sea distributions [Fig. 4(b) and 4(c)] are less precisely determined. At values of $x$ down to, say, $x \simeq 0.07$ the bands are still reasonably well confined; however, they open up widely in the small- $x$ region. Cases where the strange quark sea lies above the up-quark sea are unrealistic, but are present in some of the fits since this region $(x \lesssim 0.02)$ is not constrained by data. We have included the curves for our free-proton Base-1 PDFs (dashed line), as well as the HKN04 [3] (dotted line), the NLO HKN07 [4] (dotted-dashed line), and DS [7] (dot-dashed line) nuclear PDFs. ${ }^{15}$

The comparison with the Base-1 PDFs is straightforward since the same theoretical framework (input scale, functional form, NLO evolution) has been utilized for their determination. Therefore, the differences between the solid band and the dashed line exhibit the nuclear effects, keeping in mind that the free-proton PDFs themselves have uncertainties.

For the comparison with the HKN04 distributions, it should be noted that an SU(3)-flavor-symmetric sea has been used; therefore, the HKN04 strange quark distribution is larger, and the light quark sea smaller, than their Base-1 PDF counterparts over a wide range in $x$. Furthermore, the HKN04 PDFs are evolved at leading order.

In a recent analysis, the HKN group has published a new set of NPDFs (HKN07) including uncertainties [4]. They provide both LO and NLO sets of PDFs, and we display the NLO set. These PDFs also use a more general set of sea distributions such that $\bar{u}(x) \neq \bar{d}(x) \neq \bar{s}(x)$ in general.
The DS PDFs are linked to the GRV98 PDFs [45] with a rather small radiatively generated strange-sea distribution. Consequently, the light quark sea is enhanced compared to the other sets. Additionally, the DS sets are evolved in a 3fixed-flavor scheme in which no charm parton is included in the evolution. However, at the scale $Q=m_{c}$ of Fig. 4 this is of no importance.

\section{NUCLEAR CORRECTION FACTORS}

In the previous section we analyzed charged current $\nu$-Fe data with the goal of extracting the iron nuclear parton distribution functions. In this section, we now compare our iron PDFs with the free-proton PDFs (appropriately scaled) to infer the proper heavy target correction which should be applied to relate these quantities.

Within the parton model, a nuclear correction factor $R[\mathcal{O}]$ for an observable $\mathcal{O}$ can be defined as follows:

$$
R[\mathcal{O}]=\frac{\mathcal{O}[\mathrm{NPDF}]}{\mathcal{O}[\text { free }]}
$$

where $\mathcal{O}[\mathrm{NPDF}]$ represents the observable computed with nuclear PDFs, and $\mathcal{O}[$ free] is the same observable constructed out of the free nucleon PDFs according to Eq. (28). Clearly, $R$ can depend on the observable under consideration simply because different observables may be sensitive to different combinations of PDFs.

This means that the nuclear correction factor $R$ for $F_{2}^{A}$ and $F_{3}^{A}$ will, in general, be different. Additionally, the nuclear correction factor for $F_{2}^{A}$ will yield different results for the charged current $\nu$-Fe process ( $W^{ \pm}$exchange) as compared with the neutral current $\ell^{-} \mathrm{Fe}$ process $(\gamma$ exchange). Schematically, we can write the nuclear correction for the DIS structure function $F_{2}$ in a charged current (CC) $\nu$-A process as (cf. Eq. (7) $)^{16}$ :

$$
R_{\mathrm{CC}}^{\nu}\left(F_{2} ; x, Q^{2}\right) \simeq \frac{d^{A}+\bar{u}^{A}+\ldots}{d^{\varnothing}+\bar{u}^{\varnothing}+\ldots}
$$

and contrast this with the neutral current (NC) $\ell^{ \pm}-A$ process (cf. Eq. (14)):

$$
R_{\mathrm{NC}}^{e, \mu}\left(F_{2} ; x, Q^{2}\right) \simeq \frac{\left(-\frac{1}{3}\right)^{2}\left[d^{A}+\bar{d}^{A}+\ldots\right]+\left(+\frac{2}{3}\right)^{2}\left[u^{A}+\bar{u}^{A}+\ldots\right]}{\left(-\frac{1}{3}\right)^{2}\left[d^{\varnothing}+\bar{d}^{\varnothing}+\ldots\right]+\left(+\frac{2}{3}\right)^{2}\left[u^{\varnothing}+\bar{u}^{\varnothing}+\ldots\right]}
$$

\footnotetext{
${ }^{14}$ While iron is roughly isoscalar, other nuclear PDFs can exhibit larger differences between the $u$ and $d$ distributions - the extreme case being the free-proton PDF. When comparing PDFs of Eq. (2), we must keep in mind that it is ultimately the structure functions defined by Eq. (4) which are the physical observables.

${ }^{15}$ In a recent publication, Eskola et al. [6] perform a global reanalysis of their ESK98 [5] nuclear PDFs. While we do not present a comparison here, the results are compatible with those distributions displayed in Fig. 4; a comparison can be found in Figs. 10 and 11 of Ref. [6].

${ }^{16}$ The corresponding antineutrino process is obtained with a $u \leftrightarrow d$ interchange.
} 
where the superscript " $\varnothing$ " denotes the "free nucleon" PDF which is constructed via the relation:

$$
f_{i}^{\varnothing}(x, Q)=\frac{Z}{A} f_{i}^{p}(x, Q)+\frac{(A-Z)}{A} f_{i}^{n}(x, Q) .
$$

Clearly, the $R$-factors depend on both the kinematic variables and the factorization scale. Finally, we note that Eq. (25) is subject to uncertainties of both the numerator and the denominator.

We will now evaluate the nuclear correction factors for our extracted PDFs, and compare these with selected results from the literature [13-15]. ${ }^{17}$ Because we have extracted the iron PDFs from only iron data, we do not assume any particular form for the nuclear $A$-dependence; hence the extracted $R[\mathcal{O}]$ ratio is essentially model independent.

\section{A. Deuteron corrections for the $\boldsymbol{F}_{\mathbf{2}}^{\mathrm{Fe}} / \boldsymbol{F}_{\mathbf{2}}^{\boldsymbol{D}}$ ratio}

The structure function ratio $F_{2}^{\mathrm{Fe}} / F_{2}^{\mathrm{D}}$ provides a common (and useful) observable to use to gauge the nuclear effects of iron. To construct the numerator, we will use our iron PDFs as extracted in fits A and A2. For the denominator, we will use the Base-1 and Base-2 free-proton PDF; however, converting from free-proton structure functions to deuteron structure functions is nontrivial and model dependent.

In Fig. 5 we display the NMC data for $F_{2}^{D} / F_{2}^{p}$ [46] and compare this to a variety of data parametrizations $[9,10,46,47]$. The dashed line shows the structure function ratio computed with the Base-1 PDFs; in this case a nuclear correction factor for deuterium has been applied using the parametrization of Ref. [10]. The solid line shows the structure function ratio computed with the Base-2 PDFs; in this case no nuclear correction factor for deuterium was applied. The dotted line (Arneodo) is the parametrization of Ref. [46], and the dot-dashed line (Tvaskis) is the parametrization of Ref. [47]. We see that the range of discrepancies in the deuterium corrections are typically on the order of a percent or two except at large $x$; while this correction cannot be neglected, it is small compared to the much larger iron nuclear corrections. To explore a range of possibilities (reflecting the underlying uncertainty) we have incorporated deuteron corrections into the Base-1 PDF, but not the Base-2 PDF; hence the spread between these two reference PDFs will, in part, reflect our ignorance of $F_{2}^{D}$ and other uncertainties of proton PDFs at large $x$.

\section{B. $F_{2}^{\mathrm{Fe}} / F_{2}^{D}$ for NC charged-lepton scattering}

We will also find it instructive to compare our results with the $F_{2}^{\mathrm{Fe}} / F_{2}^{\mathrm{D}}$ as extracted in neutral current chargedlepton scattering, $\ell^{ \pm}$-Fe. In Fig. 6 we compare the experi-

\footnotetext{
${ }^{17}$ Note that our comparison with the Kulagin-Petti model is based on the work in Ref. [14].
}

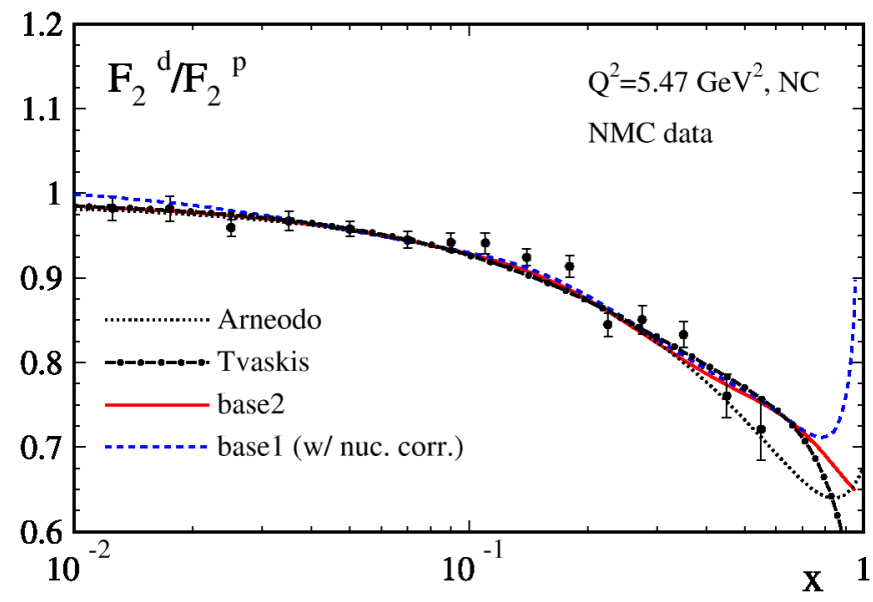

FIG. 5 (color online). NMC data for $F_{2}^{D} / F_{2}^{p}$ [46] at $Q^{2}=$ $5.47 \mathrm{GeV}^{2}$ in comparison with the theory prediction for $F_{2}^{D} / F_{2}^{p}$ computed using free-proton Base-2 PDFs. The dashed line shows the structure function ratio obtained with the Base-1 PDFs; in this case a nuclear correction factor for deuterium has been applied (cf., Refs. [9,10]). For comparison, we also show the parametrizations of Arneodo et al. [46] and Tvaskis et al. $[47,58]$.

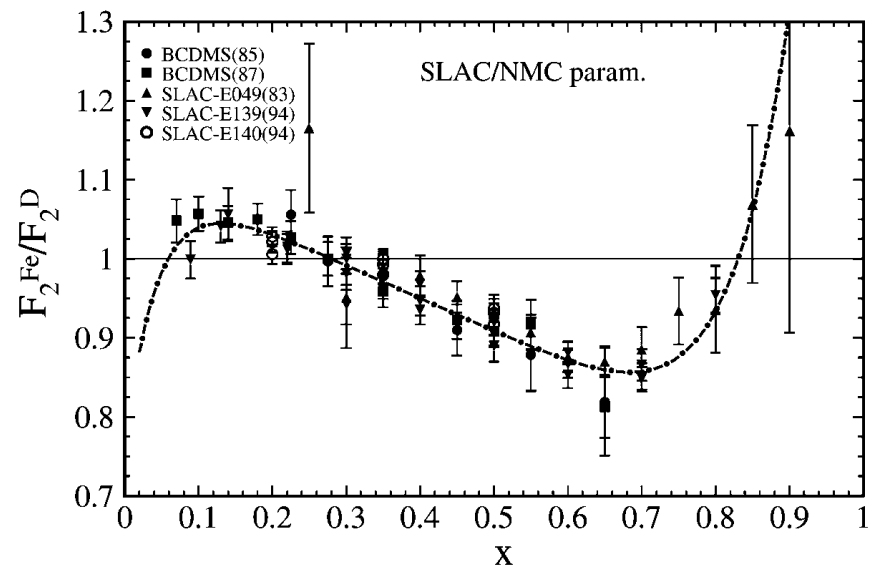

FIG. 6. Parametrization for the neutral current charged-lepton structure function $F_{2}^{\mathrm{Fe}} / F_{2}^{D}$. For comparison we show experimental results from the BCDMS collaboration (BCDMS-85 [48], BCDMS-87 [49]) and from experiments at SLAC (SLAC-E049 [50], SLAC-E139 [9], and SLAC-E140 [34]). Normalization uncertainties of the data have not been included.

mental results for the structure function ratio $F_{2}^{\mathrm{Fe}} / F_{2}^{\mathrm{D}}$ for the following experiments: BCDMS-85 [48], BCDMS-87 [49], SLAC-E049 [50], SLAC-E139 [9], SLAC-140 [34]. The curve (labeled SLAC/NMC parametrization) is a fit to this data [13]. While there is a spread in the individual data points, the parametrization describes the bulk of the data at the level of a few percent or better. It is important to note that this parametrization is independent of atomic number $A$ and the energy scale $Q^{2}$ [51]; this is in contrast to the results we will derive using the PDFs extracted from the 


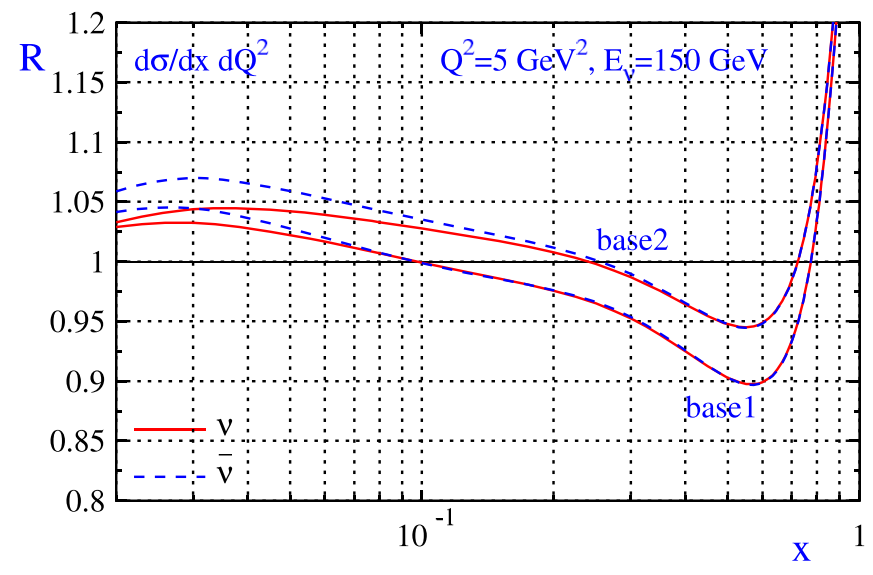

FIG. 7 (color online). Nuclear correction factor $R$ according to Eq. (25) for the differential cross section $d^{2} \sigma / d x d Q^{2}$ in charged current $\nu \mathrm{Fe}$ scattering at $Q^{2}=5 \mathrm{GeV}^{2}$ and $E_{\nu}=150 \mathrm{GeV}$. Results are shown using the A2 fit for the charged current neutrino (solid lines) and antineutrino (dashed lines) scattering from iron. The upper (lower) pair of curves shows the result of our analysis with the Base-2 (Base-1) free-proton PDFs. The correction factors shown here are for an iron target which has been corrected for the neutron excess.

nuclear data. ${ }^{18}$ Additionally, we note that while this parametrization has been extracted using ratios of $F_{2}$ structure functions, it is often applied to other observables such as $F_{1,3, L}$ or $d \sigma$. We can use this parametrization as a guide to judge the approximate correspondence between this NC charged-lepton DIS data and our CC neutrino DIS data.

\section{Correction Factors for $d^{2} \sigma / d x d Q^{2}$}

We begin by computing the nuclear correction factor $R$ according to Eq. (25) for the neutrino differential cross section in Eq. (5) as this represents the bulk of the NuTeV data included in our fit. More precisely, we show $R$-factors for the charged current cross sections $d^{2} \sigma / d x d Q^{2}$ at fixed $Q^{2}$ which can be obtained from Eq. (5) by a simple Jacobian transformation and we consider an iron target which has been corrected for the neutron excess, i.e., we use the PDFs in Eq. (2) (for the numerator) and Eq. (28) (for the denominator) with $A=56$ and $Z=28$. Our results are displayed in Fig. 7 for $Q^{2}=5 \mathrm{GeV}^{2}$ and a neutrino energy $E_{\nu}=150 \mathrm{GeV}$ which implies, due to the relation $Q^{2}=2 M E_{\nu} x y$, a minimal $x$-value of $x_{\min }=0.018$. The solid (dashed) lines correspond to neutrino (antineutrino) scattering using the iron PDFs from the A2 fit.

We have computed $R$ using both the Base- 1 and Base- 2 PDFs for the denominator of Eq. (25); recall that Base-1 includes a deuteron correction while Base-2 uses the CCFR data and does not include a deuteron correction. The

\footnotetext{
${ }^{18}$ In particular, we will find for large $x(\gtrsim 0.5)$ and $Q$ comparable to the proton mass the target mass corrections for $F_{2}^{\mathrm{Fe}} / F_{2}^{\mathrm{D}}$ are essential for reproducing the features of the data; hence the $Q$ dependence plays a fundamental role.
}

difference between the Base- 1 and Base- 2 curves is approximately $2 \%$ at small $x$ and grows to $5 \%$ at larger $x$, with Base-2 above the Base-1 results. As this behavior is typical, in the following plots (Figs. 8 and 9) we will only show the Base-1 results. We also observe that the neutrino (antineutrino) results coincide in the region of large $x$ where the valence PDFs are dominant, but differ by a few percent at small $x$ due to the differing strange and charm distributions.

\section{Correction Factors for $F_{2}^{\nu}\left(x, Q^{2}\right)$ and $F_{2}^{\bar{\nu}}\left(x, Q^{2}\right)$}

We now compute the nuclear correction factors for charged current neutrino-iron scattering. The results for $\nu$-Fe are shown in Fig. 8, and those of $\bar{\nu}$-Fe are shown in Fig. 9. The numerator in Eq. (25) has been computed using the nuclear PDF from fit A2, and for the denominator we have used the Base-1 PDFs. For comparison we also show
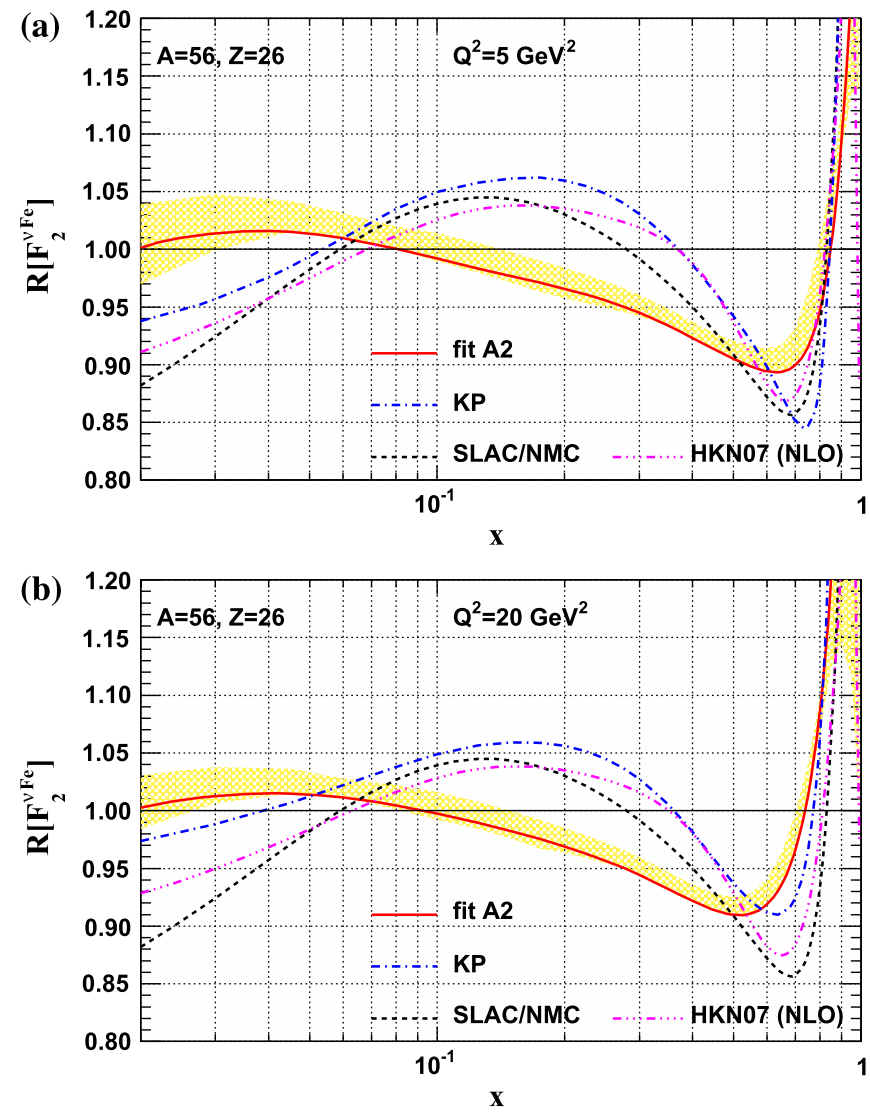

FIG. 8 (color online). Nuclear correction factor $R$ according to Eq. (25) for the structure function $F_{2}$ in charged current $\nu \mathrm{Fe}$ scattering at (a) $Q^{2}=5 \mathrm{GeV}^{2}$ and (b) $Q^{2}=20 \mathrm{GeV}^{2}$. The solid curve shows the result of our analysis of $\mathrm{NuTeV}$ data (one representative of "fit A2") divided by the results obtained with the Base-1 free-proton PDFs; the uncertainty from the A2 fit is represented by the yellow band. For comparison we show the correction factor from the Kulagin-Petti model [14] (dasheddot line), HKN07 [4] (dashed-dotted line), and the SLAC/NMC parametrization (dashed line) [13]. 

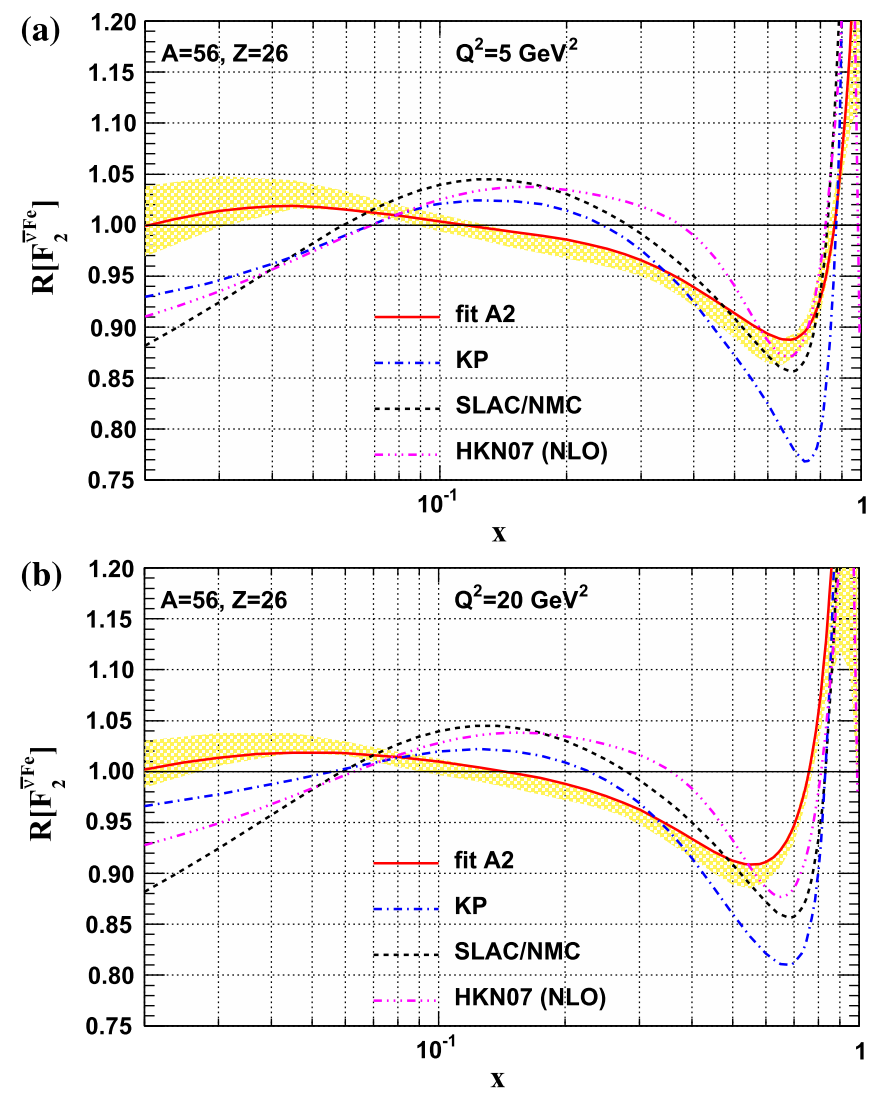

FIG. 9 (color online). The same as in Fig. 8 for $\bar{\nu}$ Fe scattering.

the correction factor from the Kulagin-Petti model [14] (dashed-dotted line), and the SLAC/NMC curve (dashed line) [13] which has been obtained from an $A$ and $Q^{2}$-independent parametrization of calcium and iron charged-lepton DIS data.

Because of the neutron excess in iron, ${ }^{19}$ both our curves and the KP curves differ when comparing scattering for neutrinos (Fig. 8) and antineutrinos (Fig. 9); the SLAC/ NMC parametrization is the same in both figures. For our results (solid lines), the difference between the neutrino and antineutrino results is relatively small, of order 3\% at $x=0.6$. Conversely, for the KP model (dashed-dotted lines) the $\nu-\bar{\nu}$ difference reaches $10 \%$ at $x \sim 0.7$, and remains sizable at lower values of $x$.

To demonstrate the dependence of the $R$ factor on the kinematic variables, in Figs. 8 and 9 we have plotted the nuclear correction factor for two separate values of $Q^{2}$. Again, our curves and the KP model yield different results

\footnotetext{
${ }^{19}$ Note that the correction factors shown in Figs. 8 and 9 are valid for the case in which the data have not been corrected for the neutron excess in iron. For data that already have been corrected for the neutron excess one should, for consistency, compute the $R$-factors using $A=56, Z=28$ in Eq. (2). The magnitude of the difference between the $R$-factors in these two cases $(Z=26$ vs $Z=28)$ is typically a few percent.
}

for different $Q^{2}$ values, in contrast to the SLAC/NMC parametrization.

Comparing the nuclear correction factors for the $F_{2}$ structure function (Figs. 8 and 9) with those obtained for the differential cross section (Fig. 7), we see these are quite different, particularly at small $x$. Again, this is because the cross section $d^{2} \sigma$ is comprised of a different combination of PDFs than the $F_{2}$ structure function. In general, our $R$-values for $F_{2}$ lie below those of the corresponding $R$-values for the cross section $d \sigma$ at small $x$. Since $d \sigma$ is a linear combination of $F_{2}$ and $F_{3}$, the $R$-values for $F_{3}$ (not shown) therefore lie above those of $F_{2}$ and $d \sigma$. Again, we emphasize that it is important to use an appropriate nuclear correction factor which is matched to the particular observable.

As we observed in the previous section, our results have general features in common with the KP model and the SLAC/NMC parametrization, but the magnitude of the effects and the $x$-region where they apply are quite different. Our results are noticeably flatter than the KP and SLAC/NMC curves, especially at moderate $-x$ where the differences are significant. The general trend we see when examining these nuclear correction factors is that the antishadowing region is shifted to smaller $x$ values and any turnover at low $x$ is minimal given the PDF uncertainties. In general, these plots suggest that the size of the nuclear corrections extracted from the $\mathrm{NuTeV}$ data are smaller than those obtained from charged-lepton scattering (SLAC/ $\mathrm{NMC}$ ) or from the set of data used in the KP model. We will investigate this difference further in the following section.

\section{E. Predictions for charged-lepton $F_{2}^{\mathrm{Fe}} / F_{2}^{D}$ from iron PDFs}

Since the SLAC/NMC parametrization was fit to $F_{2}^{\mathrm{Fe}} / F_{2}^{D}$ for charged-lepton DIS data, we can perform a more balanced comparison by using our iron PDFs to compute this same quantity. The results are shown in Fig. 10 where we have used our iron PDFs to compute $F_{2}^{\mathrm{Fe}}$, and the Base- 1 and Base-2 PDFs to compute $F_{2}^{D}$.

As with the nuclear correction factor results of the previous section, we find our results have some gross features in common while on a more refined level the magnitude of the nuclear corrections extracted from the CC iron data differs from the charged-lepton data. In particular, we note that the so-called "antishadowing" enhancement at $x \sim[0.06-0.3]$ is not reproduced by the charged current (anti)neutrino data. Examining our results among all the various $R[\mathcal{O}]$ calculations, we generally find that any nuclear enhancement in the small $x$ region is reduced and shifted to a lower $x$ range as compared with the SLAC/NMC parametrization. In fact, this behavior is expected given the comparisons of Figs. 1-3 which show that at $x \sim 0.1$ the cross sections obtained with the base $\mathrm{PDF}$ are not smaller than the A and A2 fitted cross 


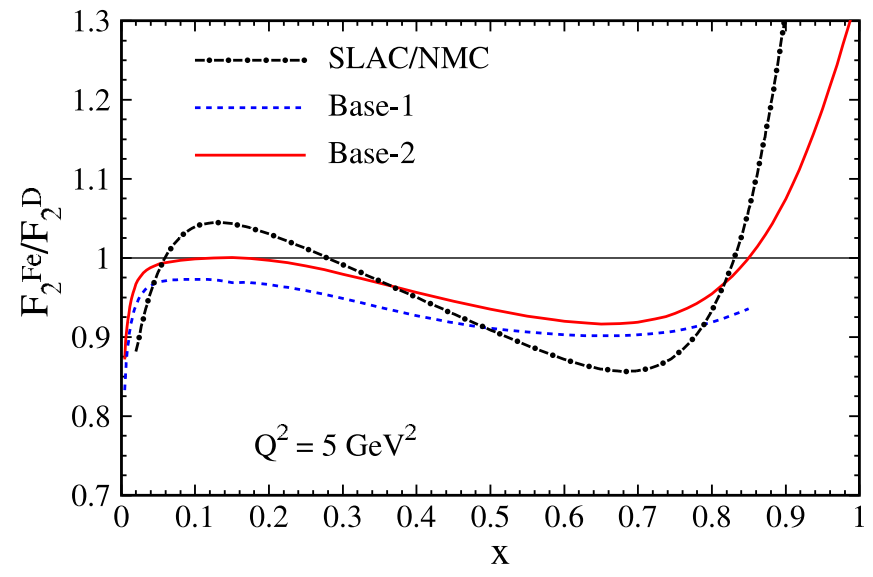

FIG. 10 (color online). Predictions (solid and dashed line) for the structure function ratio $F_{2}^{\mathrm{Fe}} / F_{2}^{D}$ using the iron PDFs extracted from fits to $\mathrm{NuTeV}$ neutrino and antineutrino data (fit A2). The SLAC/NMC parametrization is shown with the dotdashed line. The structure function $F_{2}^{D}$ in the denominator has been computed using either the Base-2 (solid line) or the Base-1 (dashed line) PDFs. A nuclear correction factor for deuterium has been included in the Base-1 calculation [10].

sections. Furthermore, in the limit of large $x(x \geq 0.6)$ our results are slightly higher than the data, including the very precise SLAC-E139 points; however, the large theoretical uncertainties on $F_{2}^{D}$ in this $x$-region (see Fig. 5) make it difficult to extract firm conclusions.

This discussion raises the more general question as to whether the charged current $(\nu$-Fe) and neutral current $\left(\ell^{ \pm}-\mathrm{Fe}\right)$ correction factors are entirely compatible $[8,44,52-55]$. There is a priori no requirement that these be equal; in fact, given that the $\nu$-Fe process involves the exchange of a $W$ and the $\ell^{ \pm}-\mathrm{Fe}$ process involves the exchange of a $\gamma$ we necessarily expect this will lead to differences at some level. To say definitively how much of this difference is due to this effect and how much is due to the uncertainty of our nuclear PDFs requires further study; in particular, it would be interesting to extend the global analysis of nuclear PDFs to include neutral current charged lepton as well as additional charged current neutrino data. Here, the analysis of additional data sets such as the ones from the CHORUS experiment [56,57] (neutrino-lead interactions) should help clarify these questions. We are in the processes of adding additional nuclear data sets to our analysis; however, this increased precision comes at the expense of introducing the A degree of freedom into the fit.

\section{CONCLUSIONS}

We have presented a detailed analysis of the high statistics NuTeV neutrino-iron data in the framework of the parton model at next-to-leading order QCD. This investigation takes a new approach to this problem by studying a single nuclear target (iron) so that we avoid the difficulty of having to assume a nuclear A dependence. In this context, we have extracted a set of iron PDFs which are free of any nuclear model dependence. By comparing these iron PDFs with "free-proton" PDFs, we can construct the associated nuclear correction factor $R$ for any chosen observable in any given $\left\{x, Q^{2}\right\}$ kinematic range.

While the nuclear corrections extracted from charged current $\nu$-Fe scattering have similar characteristics as the neutral current $\ell^{ \pm}$-Fe charged-lepton results, the detailed $x$ and $Q^{2}$ behavior is quite different. These results raise the deeper question as to whether the charged current and neutral current correction factors may be substantially different. A combined analysis of neutrino and chargedlepton data sets, for which the present study provides a foundation, will shed more light on these issues. Resolving these questions is essential if we are to reliably use the plethora of nuclear data to obtaining free-proton PDFs.

\section{ACKNOWLEDGMENTS}

We thank Tim Bolton, Javier Gomez, Shunzo Kumano, Eric Laenen, Dave Mason, W. Melnitchouk, Donna Naples, Mary Hall Reno, Voica A. Radescu, and Martin Tzanov for valuable discussions. F. I. O., I. S., and J. Y. Y. acknowledge the hospitality of Argonne, BNL, CERN, and Fermilab where a portion of this work was performed. This work was partially supported by the U.S. Department of Energy under Grant No. DE-FG02-04ER41299, Contract No. DE-AC05-06OR23177 (under which Jefferson Science Associates LLC operates the Thomas Jefferson National Accelerator Facility), the National Science Foundation Grant No. 0400332, the Lightner-Sams Foundation, and the Sam Taylor Foundation. The work of J.F. Owens was supported in part by the U.S. Department of Energy under Contract No. DE-FG02-97IR41022. The work of J.Y. Yu was supported by the Deutsche Forschungsgemeinschaft (DFG) through Grant No. YU 118/1-1.
[1] R. S. Thorne, arXiv:hep-ph/0606307.

[2] M. Hirai, S. Kumano, and M. Miyama, Phys. Rev. D 64, 034003 (2001).
[3] M. Hirai, S. Kumano, and T. H. Nagai, Phys. Rev. C 70, 044905 (2004).

[4] M. Hirai, S. Kumano, and T. H. Nagai, Phys. Rev. C 76, 
065207 (2007).

[5] K. J. Eskola, V. J. Kolhinen, and C. A. Salgado, Eur. Phys. J. C 9, 61 (1999).

[6] K. J. Eskola, V. J. Kolhinen, H. Paukkunen, and C. A. Salgado, J. High Energy Phys. 05 (2007) 002.

[7] D. de Florian and R. Sassot, Phys. Rev. D 69, 074028 (2004).

[8] M. Tzanov et al. (NuTeV Collaboration), Phys. Rev. D 74, 012008 (2006).

[9] J. Gomez et al., Phys. Rev. D 49, 4348 (1994).

[10] J. F. Owens et al., Phys. Rev. D 75, 054030 (2007).

[11] R.S. Thorne, A. D. Martin, and W. J. Stirling, arXiv:hep$\mathrm{ph} / 0606244$.

[12] J. Pumplin et al., J. High Energy Phys. 07, 012 (2002).

[13] A. Bruell (private communication).

[14] S. A. Kulagin and R. Petti, Nucl. Phys. A765, 126 (2006).

[15] S. A. Kulagin and R. Petti, Phys. Rev. D 76, 094023 (2007).

[16] I. Niculescu, J. Arrington, R. Ent, and C. E. Keppel, Phys. Rev. C 73, 045206 (2006).

[17] M. A. G. Aivazis, F. I. Olness, and W.-K. Tung, Phys. Rev. D 50, 3085 (1994).

[18] M. A. G. Aivazis, J.C. Collins, F. I. Olness, and W.-K. Tung, Phys. Rev. D 50, 3102 (1994).

[19] S. Kretzer and I. Schienbein, Phys. Rev. D 58, 094035 (1998).

[20] S. Catani, D. de Florian, G. Rodrigo, and W. Vogelsang, Phys. Rev. Lett. 93, 152003 (2004).

[21] M. Goncharov et al. (NuTeV Collaboration), Phys. Rev. D 64, 112006 (2001).

[22] D. Mason (NuTeV Collaboration), AIP Conf. Proc. 792, 851 (2005).

[23] D. Mason (NuTeV Collaboration), arXiv:hep-ex/0405037.

[24] S. Kretzer et al., Phys. Rev. Lett. 93, 041802 (2004).

[25] F. Olness et al., Eur. Phys. J. C 40, 145 (2005).

[26] S. Kretzer, D. Mason, and F. Olness, Phys. Rev. D 65, 074010 (2002).

[27] E. Oltman et al., Z. Phys. C 53, 51 (1992).

[28] W. G. Seligman et al., Phys. Rev. Lett. 79, 1213 (1997).

[29] U.-K. Yang et al. (CCFR/NuTeV Collaboration), Phys. Rev. Lett. 86, 2742 (2001).

[30] U.-K. Yang, Report No. FERMILAB-THESIS-2001-09.

[31] H. Abramowicz et al., Z. Phys. C 25, 29 (1984).

[32] J. P. Berge et al., Z. Phys. C 49, 187 (1991).

[33] J. M. Conrad, M. H. Shaevitz, and T. Bolton, Rev. Mod. Phys. 70, 1341 (1998).

[34] S. Dasu et al., Phys. Rev. D 49, 5641 (1994).
[35] J. J. Aubert et al. (European Muon Collaboration), Nucl. Phys. B272, 158 (1986).

[36] J. J. Aubert et al. (European Muon Collaboration), Nucl. Phys. B293, 740 (1987).

[37] We are grateful to $\mathrm{M}$. Tzanov for providing tables with radiative and isoscalar correction factors.

[38] S. Kretzer and M.H. Reno, Phys. Rev. D 69, 034002 (2004).

[39] I. Schienbein, V. A. Radescu, G. Zeller, M. E. Christy, C. Keppel, K. S. McFarland, W. Melnitchouk, F. I. Olness, M. H. Reno, F. Steffens, and J.-Y. Yu, arXiv:0709.1775.

[40] A. L. Kataev, A. V. Kotikov, G. Parente, and A. V. Sidorov, Phys. Lett. B 417, 374 (1998).

[41] A. L. Kataev, G. Parente, and A. V. Sidorov, arXiv:hep-ph/ 9809500.

[42] A. L. Kataev, G. Parente, and A. V. Sidorov, Nucl. Phys. B573, 405 (2000).

[43] S. I. Alekhin and A.L. Kataev, Phys. Lett. B 452, 402 (1999).

[44] M. Tzanov (NuTeV Collaboration), AIP Conf. Proc. 792, 241 (2005).

[45] M. Glück, E. Reya, and A. Vogt, Eur. Phys. J. C 5, 461 (1998).

[46] M. Arneodo et al. (New Muon Collaboration), Nucl. Phys. B487, 3 (1997).

[47] V. Tvaskis, thesis, Amsterdam University, 2004.

[48] G. Bari et al. (BCDMS Collaboration), Phys. Lett. B 163, 282 (1985).

[49] A. C. Benvenuti et al. (BCDMS Collaboration), Phys. Lett. B 189, 483 (1987).

[50] A. Bodek et al., Phys. Rev. Lett. 50, 1431 (1983).

[51] J. Arrington, R. Ent, C.E. Keppel, J. Mammei, and I. Niculescu, Phys. Rev. C 73, 035205 (2006).

[52] C. Boros, J. T. Londergan, and A. W. Thomas, Phys. Rev. Lett. 81, 4075 (1998).

[53] C. Boros, F.M. Steffens, J.T. Londergan, and A. W. Thomas, Phys. Lett. B 468, 161 (1999).

[54] A. Bodek, Q. Fan, M. Lancaster, K. S. McFarland, and U.-K. Yang, Phys. Rev. Lett. 83, 2892 (1999).

[55] S. Kretzer, F. I. Olness, R. J. Scalise, R. S. Thorne, and U.-K. Yang, Phys. Rev. D 64, 033003 (2001).

[56] G. Onengut et al. (CHORUS Collaboration), Phys. Lett. B 632, 65 (2006).

[57] A. Kayis-Topaksu et al. (CHORUS Collaboration), Eur. Phys. J. C 30, 159 (2003).

[58] V. Tvaskis et al., Phys. Rev. Lett. 98, 142301 (2007). 